Article

\title{
Dietary Flavonoids and Gastric Cancer Risk in a Korean Population
}

\section{Hae Dong Woo ${ }^{1}$, Jeonghee Lee ${ }^{1}$, Il Ju Choi ${ }^{2}$, Chan Gyoo Kim ${ }^{2}$, Jong Yeul Lee ${ }^{2}$, Oran Kwon ${ }^{3}$ and Jeongseon Kim ${ }^{1, *}$}

1 Molecular Epidemiology Branch, Division of Cancer Epidemiology and Prevention, Research Institute, National Cancer Center, Gyeonggi-do 410-769, Korea;

E-Mails: hdwoo@ncc.re.kr (H.D.W.); jeonghee@ncc.re.kr (J.L.)

2 Center for Gastric Cancer, National Cancer Center Hospital, National Cancer Center, Gyeonggi-do 410-769, Korea; E-Mails: cij1224@ncc.re.kr (I.J.C.); glse@ncc.re.kr (C.G.K.); jylee@ncc.re.kr (J.Y.L.)

3 Department of Nutritional Science and Food Management, Ewha Womans University, Seoul 120-750, Korea; E-Mail: orank@ewha.ac.kr

* Author to whom correspondence should be addressed; E-Mail: jskim@ncc.re.kr; Tel.: +82-31-920-2570; Fax: +82-31-920-2579.

Received: 4 August 2014; in revised form: 17 October 2014 / Accepted: 30 October 2014 / Published: 10 November 2014

\begin{abstract}
Gastric cancer is the most common cancer among men in Korea, and dietary factors are closely associated with gastric cancer risk. We performed a case-control study using 334 cases and 334 matched controls aged 35-75 years. Significant associations were observed in total dietary flavonoids and their subclasses, with the exception of anthocyanidins and isoflavones (OR (95\% CI): $0.49(0.31-0.76), p$ trend $=0.007$ for total flavonoids). However, these associations were not significant after further adjustment for fruits and vegetable consumption (OR (95\% CI): $0.62(0.36-1.09), p$ trend $=0.458$ for total flavonoids). Total flavonoids and their subclasses, except for isoflavones, were significantly associated with a reduced risk gastric cancer in women (OR (95\% CI): 0.33 (0.15-0.73), $p$ trend $=0.001$ for total flavonoids) but not in men (OR (95\% CI): 0.70 (0.39-1.24), $p$ trend $=0.393$ for total flavonoids). A significant inverse association with gastric cancer risk was observed in flavones, even after additional adjustment for fruits and vegetable consumption in women. No significantly different effects of flavonoids were observed between $H$. pylori-positive and negative subjects. In conclusion, dietary flavonoids were inversely associated with gastric cancer risk, and these protective effects
\end{abstract}


of dietary flavonoids were prominent in women. No clear differences were observed in the subgroup analysis of $H$. pylori and smoking status.

Keywords: gastric cancer; flavonoids; H. pylori; smoking; case-control study

\section{Introduction}

Gastric cancer is a leading cause of cancer mortality in Eastern Asia, Central and Eastern Europe, and Central and South America [1]. Gastric cancer is the most common cancer among men in Korea, and the estimated age-standardized incidence and mortality rates in 2014 were 42.1 and 8.7 per 100,000 persons, respectively [2]. Thus prevention strategies for gastric cancer are a major concern in Korea.

Diet plays an important role in gastric cancer risk. Non-starchy and allium (onion and garlic) vegetables and fruits are most likely associated with reduced gastric cancer risk, while salt and salted foods are most likely associated with an increased risk of this cancer [3]. Leafy vegetables and onions contain high amounts of flavonoids. Onion is a major source of flavonol quercetin, which has a relatively high bioavailability among dietary flavonoid components. The beneficial effects of fruits and vegetables on gastric cancer could be related to flavonoid intake as well as other phytochemicals. We performed a meta-analysis to investigate the effects of dietary flavonoids on gastric cancer risk in a previous study. Total flavonoids and their subclasses except flavonols and quercetin were not significantly associated with a reduced risk of gastric cancer [4]. However, only a small number of papers that reported the effects of dietary flavonoids on gastric cancer risk have been published, and the reported flavonoid subclasses differed across studies. We could not confirm a clear link between dietary flavonoids and gastric cancer risk in the meta-analysis of [5-10]. Thus, additional data should be collected to identify the true associations.

Helicobacter pylori (H. pylori) and smoking have been classified as causes of cancer of the stomach, according to a monograph from the International Agency for Research on Cancer (IARC) [11,12]. $H$. pylori infection is a major risk factor for gastric cancer, and the growth of $H$. pylori infection was inhibited by flavonoids in an in vitro study. Thus, $H$. pylori-induced gastric cancer might be prevented by an appropriate diet. In previous meta-analyses, dietary flavonoids were inversely associated with smoking-related cancer [13] because tobacco contains various carcinogens that can induce free radicals and cause gene mutations in the formation of DNA [14]. The beneficial effects of flavonoids on human health could be from antioxidant activities or regulation of enzymatic pathways. The protective effects of flavonoids on human health cannot be accounted for only by direct antioxidant activities due to low bioavailability. Flavonoids are responsible for the inactivation of phase I enzymes, as well as the activation of phase II enzymes [15]. Thus, dietary flavonoids may play a role in protecting against carcinogens.

Thus, we performed a case-control study to investigate the effects of dietary flavonoids and their subclasses on gastric cancer risk. We included subgroup analyses by $H$. pylori infection and smoking status. 


\section{Experimental Section}

\subsection{Study Population}

Cases were defined as histologically confirmed early gastric cancer patients within three months of being recruited at the Center for Gastric Cancer in the National Cancer Center hospital in Korea between April 2011 and May 2014. Patients with diabetes mellitus, other cancer diagnosed within five years, advanced gastric cancer, severe systemic or mental disease, and women who were pregnant and breastfeeding were excluded. Among the 455 subjects who agreed to participate in the study, patients with daily energy intakes of $<500 \mathrm{kcal}$ or $>5000 \mathrm{kcal}(n=3)$ and those with missing information regarding $H$. pylori $(n=5)$ or smoking status $(n=3)$ were excluded. Controls were recruited from individuals who underwent health-screening examinations at the Center for Cancer Prevention and Detection at the same hospital during the same period. Visitors are beneficiaries of the National Health Insurance program; nearly all Koreans are the beneficiaries of this program. People with cancer, diabetes mellitus, gastric or duodenal ulcer, and previous $H$. pylori treatment were excluded. Among the 899 subjects who agreed to participate in the study, subjects with implausible energy intake $(n=6)$, and missing information in $H$. pylori $(n=13)$ and smoking status $(n=4)$ were excluded. Cases and controls were matched by sex, education (<middle school, high school, and $\geq$ college), and into 10-year age groups. A total of 334 cases and 334 matched controls were finally selected for this study. All participants were provided a written informed consent form, and the study protocol was approved by the Institutional Review Board of the National Cancer Center (IRB protocol number NCCNCS 11-438).

\subsection{Data Collection and Dietary Assessment}

The participants were asked to complete a self-administered questionnaire, which was used to gather information on demographics, lifestyle, and the medical history of the participants. Participants were asked about the average frequency of intake and portion size of specific foods to assess their regular intake during the previous year (i.e., last 12 months) using the validated food frequency questionnaire (FFQ) covering 103 food items [16]. The detailed procedure to design the FFQ is described by Ahn et al. [17]. Nine categories of frequency (never or rarely, once a month, two or three times a month, once or twice a week, three or four times a week, five or six times a week, once a day, twice a day, and three times a day) and three portion sizes (small, medium, and large) were specified on the FFQ. After collecting the dietary data, we calculated the individual food intake using CAN-PRO 3.0 (Computer Aided Nutritional Analysis Program, The Korean Nutrition Society, Seoul, Korea). The intake of flavonols, flavones, flavanones, flavan-3-ols, anthocyanidins, and isoflavones were estimated on the basis of the flavonoid database developed by Yang et al. [18,19]. Briefly, the flavonoid database was constructed using the United States Department of Agriculture (USDA) flavonoid database, the Korea functional food composition table, and the Japan functional food factor database. Foods that do not have flavonoid contents in those databases were additionally searched for in published papers. Intake of flavonols was the sum of isorhamnetin, kaempferol, myricetin, and quercetin; intake of flavones was the sum of apigenin, and luteolin; intake of flavanones was the sum of eriodictyol, hesperetin, and naringenin; intake of flavan-3-ols was the sum of catechin, epigallocatechin, epicatechin, epicatechin 3-gallate, epigallocatechin 3-gallate, gallocatechin, catechin 3-gallate 
theaflavin, thearubigins, theaflavin-3,3-digallate, theaflavin-3-gallate, and theaflavin-3'-gallate; intake of anthocyanidins was the sum of cyanidinn, delphinidin, malvidin, pelargonidin, peonidin, and petunidin; and intake of isoflavones was the sum of daidzein, genistein, and glycitein. Total flavonoid intake was the sum of the above flavonoid subclasses. A total of 418 food items from our food consumption data were matched to the flavonoid database. Except for meats, eggs, seafood, and dairy products, which are known to barely contain flavonoid (139 food items), the flavonoid database covered $98 \%$ of the consumed food weight of our data. The validity of the FFQ for flavonoid intake has been tested using the three-day dietary record as a gold standard in a total of 202 persons. The crude, energy-adjusted, and de-attenuated energy-adjusted correlation coefficients for total flavonoids were $0.36,0.25$, and 0.32 , respectively. $H$. pylori infection was evaluated using a rapid urease test according to the manufacturer's instructions (Pronto Dry; Medical Instruments Corporation, Solothurn, Switzerland) and by histological evaluation.

\subsection{Statistical Analysis}

Statistical analysis was performed using the SAS version 9.2 statistical package (SAS Institute Inc., Cary, NC, USA). Using a Student $t$-test for continuous variables and a chi-square test for categorical variables, we compared the general characteristics between the case and control subjects. Total flavonoids and their subclasses were adjusted for total energy intake by the regression residual method [20]. Residuals of total flavonoids and flavonoid subclasses were categorized by tertiles, based on the control distribution for the analysis. Odds ratios and 95\% confidence intervals for gastric cancer risk were calculated across the tertiles of dietary flavonoids using logistic regression after controlling for known risk factors. The lowest tertile of each dietary flavonoid was used as the reference. Tests for trends across tertiles were performed via the Wald test using continuous variables for each dietary flavonoid (energy-adjusted residual). Model 1 was adjusted for age, total energy intake, $H$. pylori status, occupation, smoking status, alcohol consumption status, BMI, physical activity, and consumption of pickled vegetables and red and processed meat. Model 2 was further adjusted for consumption of fruits and vegetables. Subclass analyses were performed after stratification by H. pylori and smoking status.

\section{Results}

The general characteristics of the study participants are reported in Table 1. Cases had a higher total energy intake $(p<0.001)$ and a higher rate of $H$. pylori infection $(p<0.001)$, and they were more likely to be current smokers $(p<0.018)$. No differences were observed between cases and controls with respect to alcohol intake or occupational status.

The ORs and 95\% CIs of gastric cancer were analyzed across the tertiles of dietary flavonoids and their subclasses (Table 2). Significant associations were observed in total dietary flavonoids and their subclasses, with the exception of anthocyanidins and isoflavones [OR (95\% CI): 0.49 (0.31-0.76), $p$ trend $=0.007$ for total flavonoids]. However, these associations were not significant after further adjustment for fruits and vegetable consumption [OR (95\% CI): $0.62(0.36-1.09), p$ trend $=0.458$ for total flavonoids]. Total flavonoids and their subclasses, except for isoflavones, were significantly associated with a reduced risk gastric cancer in women [OR (95\% CI): $0.33(0.15-0.73), p$ trend $=0.001$ 
for total flavonoids] but not in men [OR (95\% CI): $0.70(0.39-1.24), p$ trend $=0.393$ for total flavonoids]. After additional adjustment for fruits and vegetables or vitamin $C$, none of the associations were significant. In females, gastric cancer risk was significantly associated with flavones after adjustment for fruits and vegetables, and with flavones and flavanones after adjustment for vitamin C (data not shown). Vitamin C and flavonoids were highly correlated each other, and this lead to high multicollinearity.

Table 1. General characteristics of the study subjects.

\begin{tabular}{|c|c|c|c|}
\hline & Controls $(n=334)$ & Cases $(n=334)$ & $\boldsymbol{P}$ \\
\hline Age (y) & $51.8 \pm 7.6$ & $51.8 \pm 9.0$ & 0.996 \\
\hline Male, $n(\%)$ & $208(62.3)$ & $208(62.3)$ & - \\
\hline Total energy intake (kcal) & $1817.7 \pm 618.1$ & $2010.2 \pm 649.1$ & $<0.001$ \\
\hline Physical activity (MET-min/week) & $3550.21 \pm 4756.69$ & $3429.89 \pm 5576.47$ & 0.047 \\
\hline $\mathrm{BMI}\left(\mathrm{Kg} / \mathrm{m}^{2}\right)$ & $24.1 \pm 2.9$ & $23.9 \pm 3.1$ & 0.210 \\
\hline H. pylori infection & & & $<0.001$ \\
\hline Negative & 159 (47.6) & $52(15.6)$ & \\
\hline Positive & $175(52.4)$ & $282(84.4)$ & \\
\hline Smoking status, $n(\%)$ & & & 0.018 \\
\hline Current & $71(21.3)$ & $103(30.8)$ & \\
\hline Former & $104(31.1)$ & $89(26.7)$ & \\
\hline Never & $159(47.6)$ & $142(42.5)$ & \\
\hline Alcohol intake & & & 0.262 \\
\hline Current & $214(64.1)$ & $208(62.3)$ & \\
\hline Former & $19(5.7)$ & $30(9.0)$ & \\
\hline Never & $101(30.2)$ & $96(28.7)$ & \\
\hline Education, $n(\%)$ & & & - \\
\hline$<$ Middle school & $93(27.8)$ & $93(27.8)$ & \\
\hline High school & 146 (43.7) & $146(43.7)$ & \\
\hline$\geq$ College & $95(28.4)$ & $95(28.4)$ & \\
\hline Occupation, $n(\%)$ & & & 0.208 \\
\hline Professional & $52(15.6)$ & $62(18.6)$ & \\
\hline Office/service worker & $133(39.8)$ & $107(32.0)$ & \\
\hline Manual worker & $63(18.9)$ & $68(20.4)$ & \\
\hline Other & $86(25.8)$ & $97(29.0)$ & \\
\hline \multicolumn{4}{|l|}{ Dietary flavonoids (mg/day) } \\
\hline Total flavonoids & $106.4 \pm 82.2$ & $105.2 \pm 77.9$ & $0.008^{\dagger}$ \\
\hline Flavonols & $22.8 \pm 19.5$ & $23.3 \pm 21.4$ & $0.042^{\dagger}$ \\
\hline Flavones & $1.4 \pm 1.1$ & $1.3 \pm 1.0$ & $0.021^{\dagger}$ \\
\hline Flavanones & $6.4 \pm 10.0$ & $5.8 \pm 9.5$ & $0.005^{\dagger}$ \\
\hline Flavan-3-ols & $22.8 \pm 39.2$ & $20.0 \pm 35.2$ & $<0.001^{\dagger}$ \\
\hline Anthocyanidins & $23.6 \pm 25.0$ & $24.9 \pm 22.9$ & $0.194^{\dagger}$ \\
\hline Isoflavones & $29.4 \pm 27.8$ & $29.8 \pm 25.0$ & $0.158^{\dagger}$ \\
\hline
\end{tabular}

${ }^{\dagger}$ Test was performed using each energy-adjusted nutrient residual. 
Table 2. ORs and 95\% CIs of gastric cancer by tertiles of dietary flavonoids.

\begin{tabular}{|c|c|c|c|c|c|c|c|c|c|}
\hline \multirow{2}{*}{$\begin{array}{c}\text { Tertiles of Energy } \\
\text { Adjusted Flavonoid } \\
\text { Intake (mg/d) }{ }^{\dagger}\end{array}$} & \multicolumn{3}{|c|}{ Total $(n=668)$} & \multicolumn{3}{|c|}{$\operatorname{Men}(n=416)$} & \multicolumn{3}{|c|}{ Women $(n=252)$} \\
\hline & $\begin{array}{l}\text { Controls/ } \\
\text { Cases } \\
\end{array}$ & $\begin{array}{l}\text { Model } 1 \\
\text { OR (95\% CI) } \\
\end{array}$ & $\begin{array}{l}\text { Model } 2 \\
\text { OR (95\% CI) } \\
\end{array}$ & $\begin{array}{l}\text { Controls/ } \\
\text { Cases } \\
\end{array}$ & $\begin{array}{l}\text { Model } 1 \\
\text { OR (95\% CI) } \\
\end{array}$ & $\begin{array}{l}\text { Model } 2 \\
\text { OR (95\% CI) } \\
\end{array}$ & $\begin{array}{l}\text { Controls/ } \\
\text { Cases } \\
\end{array}$ & $\begin{array}{l}\text { Model } 1 \\
\text { OR (95\% CI) } \\
\end{array}$ & $\begin{array}{l}\text { Model } 2 \\
\text { OR (95\% CI) } \\
\end{array}$ \\
\hline \multicolumn{10}{|l|}{ Total flavonoids (median) } \\
\hline $\mathrm{T} 1$ (52.5) & $111 / 152$ & 1 & 1 & 80/99 & 1 & 1 & $31 / 53$ & 1 & 1 \\
\hline $\mathrm{T} 2$ (73.2) & $111 / 94$ & $0.55(0.36-0.83)$ & $0.62(0.39-0.98)$ & $66 / 61$ & $0.75(0.44-1.29)$ & $0.80(0.45-1.43)$ & $45 / 33$ & $0.49(0.23-1.05)$ & $0.68(0.30-1.53)$ \\
\hline Т3 (152.3) & $112 / 88$ & $0.49(0.31-0.76)$ & $0.62(0.36-1.09)$ & $62 / 48$ & $0.70(0.39-1.24)$ & $0.80(0.39-1.63)$ & $50 / 40$ & $0.33(0.15-0.73)$ & $0.68(0.25-1.86)$ \\
\hline$p$ for trend ${ }^{\ddagger}$ & & 0.007 & 0.458 & & 0.393 & 0.977 & & 0.001 & 0.243 \\
\hline \multicolumn{10}{|l|}{ Flavonols (median) } \\
\hline $\mathrm{T} 1$ (10.9) & $112 / 134$ & 1 & 1 & 79/91 & 1 & 1 & $33 / 43$ & 1 & 1 \\
\hline T2 (14.4) & $111 / 114$ & $0.73(0.48-1.11)$ & $0.86(0.55-1.36)$ & $67 / 74$ & $0.84(0.49-1.44)$ & $0.89(0.49-1.61)$ & $44 / 40$ & $0.55(0.26-1.20)$ & $0.85(0.37-1.97)$ \\
\hline Т3 (30.8) & $111 / 86$ & $0.51(0.32-0.82)$ & $0.69(0.39-1.20)$ & $62 / 43$ & $0.59(0.32-1.10)$ & $0.65(0.32-1.35)$ & $49 / 43$ & $0.51(0.24-1.10)$ & $1.22(0.47-3.16)$ \\
\hline$p$ for trend & & 0.014 & 0.543 & & 0.264 & 0.585 & & 0.017 & 0.987 \\
\hline \multicolumn{10}{|l|}{ Flavones (median) } \\
\hline $\mathrm{T} 1(0.6)$ & $111 / 136$ & 1 & 1 & $85 / 93$ & 1 & 1 & $26 / 43$ & 1 & 1 \\
\hline $\mathrm{T} 2(1.0)$ & $112 / 110$ & $0.69(0.45-1.06)$ & $0.82(0.51-1.32)$ & $70 / 69$ & $0.85(0.49-1.47)$ & $0.93(0.50-1.72)$ & $42 / 41$ & $0.36(0.15-0.85)$ & $0.42(0.17-1.03)$ \\
\hline T3 (2.1) & $111 / 88$ & $0.51(0.31-0.82)$ & $0.72(0.38-1.35)$ & $53 / 46$ & $0.70(0.38-1.29)$ & $0.84(0.37-1.89)$ & $58 / 42$ & $0.15(0.06-0.38)$ & $0.22(0.07-0.67)$ \\
\hline$p$ for trend & & 0.004 & 0.411 & & 0.690 & 0.385 & & $<.0001$ & 0.010 \\
\hline \multicolumn{10}{|l|}{ Flavanones (median) } \\
\hline $\mathrm{T} 1(0.7)$ & $111 / 133$ & 1 & 1 & $88 / 94$ & 1 & 1 & 23/39 & 1 & 1 \\
\hline T2 (3.5) & $112 / 113$ & $0.85(0.56-1.28)$ & $0.98(0.64-1.50)$ & $74 / 71$ & $0.88(0.54-1.44)$ & $0.98(0.58-1.64)$ & $38 / 42$ & $0.77(0.33-1.81)$ & $0.90(0.37-2.15)$ \\
\hline T3 (11.0) & $111 / 88$ & $0.66(0.43-1.02)$ & $0.92(0.55-1.52)$ & $46 / 43$ & $0.90(0.52-1.56)$ & $1.12(0.58-2.17)$ & $65 / 45$ & $0.39(0.18-0.86)$ & $0.64(0.27-1.52)$ \\
\hline$p$ for trend & & 0.032 & 0.506 & & 0.476 & 0.987 & & 0.001 & 0.054 \\
\hline \multicolumn{10}{|l|}{ Flavan-3-ols (median) } \\
\hline $\mathrm{T} 1$ (3.2) & 111/155 & 1 & 1 & $82 / 107$ & 1 & 1 & $29 / 48$ & 1 & 1 \\
\hline $\mathrm{T} 2$ (10.8) & $111 / 90$ & $0.61(0.40-0.92)$ & $0.71(0.45-1.11)$ & $67 / 53$ & $0.76(0.44-1.30)$ & $0.82(0.45-1.48)$ & $44 / 37$ & $0.43(0.20-0.95)$ & $0.62(0.27-1.44)$ \\
\hline Т3 (39.7) & $112 / 89$ & $0.58(0.38-0.88)$ & $0.73(0.45-1.18)$ & $59 / 48$ & $0.70(0.41-1.21)$ & $0.78(0.41-1.49)$ & $53 / 41$ & $0.36(0.17-0.77)$ & $0.65(0.27-1.57)$ \\
\hline$p$ for trend & & 0.001 & 0.066 & & 0.062 & 0.175 & & 0.004 & 0.169 \\
\hline
\end{tabular}


Table 2. Cont.

\begin{tabular}{|c|c|c|c|c|c|c|c|c|c|}
\hline \multicolumn{10}{|c|}{ Anthocyanidins (median) } \\
\hline $\mathrm{T} 1(9.4)$ & $111 / 130$ & 1 & 1 & $76 / 79$ & 1 & 1 & $35 / 51$ & 1 & 1 \\
\hline T2 (14.7) & 112/99 & $0.68(0.45-1.04)$ & $0.86(0.54-1.35)$ & $65 / 65$ & $0.86(0.50-1.48)$ & $1.01(0.55-1.83)$ & $47 / 34$ & $0.53(0.26-1.10)$ & $0.80(0.37-1.76)$ \\
\hline T3 (35.1) & $111 / 105$ & $0.73(0.46-1.15)$ & $1.06(0.62-1.80)$ & $67 / 64$ & $0.92(0.51-1.67)$ & $1.16(0.57-2.34)$ & $44 / 41$ & $0.58(0.27-1.25)$ & $1.22(0.49-3.01)$ \\
\hline$p$ for trend & & 0.065 & 0.976 & & 0.972 & 0.419 & & 0.005 & 0.306 \\
\hline \multicolumn{10}{|c|}{ Isoflavones (median) } \\
\hline $\mathrm{T} 1(11.4)$ & $111 / 110$ & 1 & 1 & $78 / 67$ & 1 & 1 & $33 / 43$ & 1 & 1 \\
\hline T2 (19.9) & $112 / 144$ & $1.29(0.86-1.94)$ & $1.42(0.94-2.15)$ & $66 / 98$ & $1.90(1.12-3.21)$ & 1.99 (1.17-3.38) & $46 / 46$ & $0.64(0.31-1.34)$ & $0.76(0.35-1.63)$ \\
\hline T3 (43.7) & $111 / 80$ & $0.72(0.46-1.12)$ & $0.85(0.54-1.35)$ & $64 / 43$ & $0.90(0.52-1.54)$ & $0.98(0.56-1.73)$ & $47 / 37$ & $0.51(0.24-1.08)$ & $0.67(0.31-1.47)$ \\
\hline$p$ for trend & & 0.115 & 0.400 & & 0.494 & 0.694 & & 0.059 & 0.259 \\
\hline
\end{tabular}

${ }^{\dagger}$ Data were categorized by tertiles of each energy-adjusted dietary flavonoids based on the distribution in controls. ${ }^{\ddagger}$ Tests for trend across tertiles were performed by Wald test using continuous variables of each dietary flavonoid (energy-adjusted residual). Model 1: adjusted for total energy intake, H. pylori, age, sex, education, smoking status, alcohol consumption, BMI, physical activity, and consumption of pickled vegetable and red and processed meat. Model 2: additional adjustment for fruits and vegetable consumption. 
Table 3. ORs and 95\% CIs of gastric cancer by tertiles of dietary flavonoids stratified by H. pylori status.

\begin{tabular}{|c|c|c|c|c|c|c|c|c|}
\hline & \multicolumn{4}{|c|}{ H. pylori Positive } & \multicolumn{4}{|c|}{ H. pylori Negative } \\
\hline & $\operatorname{Men}(n=290)$ & $p$ for trend $^{\dagger}$ & Women $(n=167)$ & $p$ for trend & $\operatorname{Men}(n=126)$ & $p$ for trend & Women $(n=85)$ & $p$ for trend \\
\hline \multicolumn{9}{|l|}{ Total flavonoids (median) } \\
\hline Model 1 OR (95\% CI) & $0.50(0.25-1.01)$ & 0.193 & $0.31(0.11-0.83)$ & 0.005 & $1.32(0.41-4.24)$ & 0.996 & $0.13(0.02-0.74)$ & 0.022 \\
\hline Model 2 OR (95\% CI) & $0.57(0.24-1.33)$ & 0.733 & $0.78(0.22-2.84)$ & 0.454 & $1.37(0.32-5.76)$ & 0.889 & $0.30(0.04-2.17)$ & 0.205 \\
\hline \multicolumn{9}{|l|}{ Flavonols (median) } \\
\hline Model 1 OR (95\% CI) & $0.55(0.26-1.17)$ & 0.098 & $0.48(0.19-1.21)$ & 0.032 & $0.61(0.19-2.01)$ & 0.747 & $0.34(0.06-1.81)$ & 0.182 \\
\hline Model 2 OR (95\% CI) & $0.67(0.28-1.62)$ & 0.386 & $1.53(0.45-5.18)$ & 0.812 & $0.50(0.13-1.98)$ & 0.828 & $0.89(0.13-6.15)$ & 0.719 \\
\hline \multicolumn{9}{|l|}{ Flavones (median) } \\
\hline Model 1 OR (95\% CI) & $0.71(0.34-1.47)$ & 0.769 & $0.12(0.04-0.40)$ & $<0.001$ & $0.57(0.14-2.34)$ & 0.678 & $0.07(0.01-0.47)$ & 0.005 \\
\hline Model 2 OR (95\% CI) & $1.05(0.40-2.78)$ & 0.201 & $0.20(0.05-0.87)$ & 0.025 & $0.38(0.07-2.13)$ & 0.527 & $0.12(0.01-1.06)$ & 0.056 \\
\hline \multicolumn{9}{|l|}{ Flavanones (median) } \\
\hline Model 1 OR (95\% CI) & $0.99(0.51-1.94)$ & 0.784 & $0.36(0.14-0.91)$ & 0.003 & $0.80(0.25-2.53)$ & 0.242 & $0.22(0.03-1.44)$ & 0.010 \\
\hline Model 2 OR (95\% CI) & $1.45(0.65-3.24)$ & 0.484 & $0.62(0.22-1.72)$ & 0.059 & $0.68(0.17-2.68)$ & 0.207 & $0.52(0.06-4.38)$ & 0.088 \\
\hline \multicolumn{9}{|l|}{ Flavan-3-ols (median) } \\
\hline Model 1 OR (95\% CI) & $0.66(0.35-1.25)$ & 0.132 & $0.34(0.14-0.84)$ & 0.007 & $0.70(0.22-2.19)$ & 0.113 & $0.11(0.02-0.80)$ & 0.050 \\
\hline Model 2 OR (95\% CI) & $0.80(0.38-1.72)$ & 0.439 & $0.64(0.23-1.82)$ & 0.207 & $0.58(0.15-2.25)$ & 0.095 & $0.28(0.03-2.55)$ & 0.315 \\
\hline \multicolumn{9}{|l|}{ Anthocyanidins (median) } \\
\hline Model 1 OR (95\% CI) & $0.85(0.41-1.75)$ & 0.524 & $0.85(0.34-2.15)$ & 0.071 & $1.05(0.32-3.45)$ & 0.305 & $0.04(0.00-0.42)$ & 0.004 \\
\hline Model 2 OR (95\% CI) & $1.18(0.51-2.71)$ & 0.720 & $2.55(0.80-8.12)$ & 0.937 & $1.02(0.23-4.52)$ & 0.310 & $0.06(0.01-0.69)$ & 0.032 \\
\hline \multicolumn{9}{|l|}{ Isoflavones (median) } \\
\hline Model 1 OR (95\% CI) & $0.77(0.41-1.44)$ & 0.199 & $0.48(0.19-1.18)$ & 0.138 & $1.12(0.35-3.55)$ & 0.631 & $0.50(0.11-2.17)$ & 0.341 \\
\hline Model 2 OR (95\% CI) & $0.85(0.45-1.63)$ & 0.338 & $0.65(0.25-1.69)$ & 0.502 & $1.15(0.33-4.01)$ & 0.674 & $0.72(0.15-3.41)$ & 0.597 \\
\hline
\end{tabular}

${ }^{\dagger}$ Tests for trend across tertiles were performed by Wald test using continuous variables of each dietary flavonoid (energy-adjusted residual). Model 1: adjusted for total energy intake, age, education, smoking status, alcohol consumption, BMI, physical activity, and consumption of pickled vegetable and red and processed meat. Model 2: additional adjustment for fruits and vegetable consumption. 
The associations between dietary flavonoid intake and gastric cancer risk were analyzed after stratification by $H$. pylori (Table 3). No clear differences in the overall effects of dietary flavonoid on gastric cancer risk were found for $H$. pylori infection status (Table 3 ). $H$. pylori-positive subjects in the highest tertile of total flavonoid and flavan-3-ol consumption were associated with reduced risk in both models, and $H$. pylori-negative subjects in the highest tertile of total flavonoid and flavone consumption were associated with a reduced risk of gastric cancer in the multivariate model. However, the tertile trends for most flavonoids were not significant in either $H$. pylori-positive and negative subjects, except for flavones. Current smokers, former smokers, and never smokers were only compared among male subjects because very few women were ever smokers; the association between flavonoid intake and gastric cancer risk was not significantly different by smoking status (data not shown).

\section{Discussion}

Total dietary flavonoid intake was associated with a reduced risk of stomach cancer, especially among female subjects. However, significant associations were no longer observed except for flavones after adjustment of fruits and vegetable consumption. No clear differences were observed in the subgroup analysis of $H$. pylori.

Our previous meta-analysis, despite the limited number of studies, showed that total flavonoids and their subclasses, except flavonols and quercetin, were not significantly associated with gastric cancer risk [4]. In previous studies, flavonols including the major component quercetin have been shown to be significantly associated with reduced risk of various cancer types. Particularly, due to high bioavailability of quercetin, it has been examined in many cancer studies compared to other sub-classes of flavonoids. In the present study, most flavonoid subclasses were associated with a reduced risk of gastric cancer except for anthocyanidins and isoflavones. The main source of flavonoids could differ according to the food culture of the study subjects. In the present study, the main sources of total flavonoids were green tea, tofu, and black soybeans; flavonols came from onions and radish leaves; flavones were from mandarins and cabbage kimchi; flavanones were from mandarins and orange juice; flavan-3-ols were primarily from green tea; anthocyanidins were from radish root and radish root kimchi; and isoflavones were from tofu and black soybeans. However, the food sources of flavonols, anthocyanidins, and isoflavones were primarily kimchi or fermented soybean paste stew, which have high salt contents. Previous studies [21,22] demonstrated that fermented soy paste and pickled vegetables were associated with increased gastric cancer risk due to high salt intake. Although intake of pickled vegetables was adjusted in the analysis of the models, high intake of salts could negatively affect the beneficial effect of dietary flavonoids. We have included the fruit and vegetable consumption in the model to compare the results with/without adjustment of the variable. Flavonoids and subclasses, with exception of flavones in female, were not significantly associated with gastric cancer after adjustment of fruits and vegetable consumption. Thus, beneficial effects of flavonoids might be closely associated with fruits and vegetable consumption.

In this study, the associations between flavonoids and gastric cancer risk were only significant in the female group. Similarly, the effects of dietary flavonoids on gastric cancer risk were more prominent in female in previous studies. In addition, total dietary flavonoid intake was significantly associated with reduced gastric cancer risk in female but not in male [5], and the strong protective 
effect of quercetin was also observed in female smokers [6]. In a review of green tea consumption, green tea was not inversely associated with gastric cancer overall, but it was suggested that green tea might be associated with a reduction in gastric cancer risk among women [23]. It was also suggested that men and women have different mechanisms in redox systems [24,25]. Smoking is classified as a cause of gastric cancer [12]; thus, the effect of flavonoids on the smoker group with respect to developing gastric cancer could differ by sex. However, the different effects of flavonoids in women could not be tested because smoking rates were very low among women in this study. The effects of flavonoids on gastric cancer were not significantly different in men by smoking status (data not shown). Growth of $H$. pylori infection was inhibited by flavonoids from licorice extract in an in vitro study [26], and $H$. pylori reduces vitamin C bioavailability. Thus, it was expected that dietary flavonoids were differentially associated with gastric cancer risk based on $H$. pylori infection status. However, the associations between flavonoids and gastric cancer risk were not significantly different by $H$. pylori infection status, and these associations did not differ by sex. The sex differences in the effects of flavonoid intake in this study may not be primarily caused by smoking or $H$. pylori infection. The absolute amount of the intake of most flavonoids was higher in women, and energy-adjusted flavonoid intakes were significantly higher in women than men in the present study. In addition, food sources of flavonoids were slightly different between men and women. Flavonoids consumed from pickled vegetables were considerably higher in men, and fruit consumption was lower in men than in women. Thus, different effects of dietary flavonoids on gastric cancer in men and women may be caused by the amount of flavonoid intake and the food sources of flavonoids.

Previous Korean studies investigated the association between dietary flavonoids and disease and found that flavan3-ol intake was inversely related to the risk of metabolic syndrome in women but not in men. However, flavan-3-ol intake was protective against hypertension in non-obese men [19]. The highest tertile of flavan-3-ols exhibited significantly lowered gastric cancer risk compared with the lowest tertile, but linear trends were not observed in either men or women in this study. Serum isoflavones were inversely associated with gastric cancer risk in the Korean population [27] and inhibit cancer cell growth through activation of a signal transduction pathway for apoptosis in an in vitro study [28]. However, isoflavone intake was not significantly associated with gastric cancer risk in this study.

The results need to be interpreted with caution due to several limitations. Proanthocyanidins, an abundant group of flavonoids, were not included in total flavonoids database. Bias might be introduced because it was a case-control study. Flavonoid contents in food consumed by the study population might be different from those denoted in the database. The effect of dietary flavonoids cannot be clearly distinguished from that of phytochemicals, such as vitamin C, because they are highly correlated with each other. The bioavailability of each food is different; thus, actual uptake of flavonoids might not be equal.

\section{Conclusions}

The protective effects of dietary flavonoids were prominent in women. A clear decreasing trend in gastric cancer risk was observed to be associated with an increase in the dietary intake of total flavonoids, flavones, and anthocyanidins among female subjects. No clear differences were observed in the subgroup analysis of $H$. pylori and smoking status. 


\section{Acknowledgments}

This work was supported by the grant from the National Cancer Center, Republic of Korea (no. 1110300, 1310280, and 1410260).

\section{Author Contributions}

HDW and JK: analyzed and interpreted the data and wrote the manuscript; JL: conducted research; IJC, CGK, JYL: contributed to data collection; OK: provided the flavonoid database; and JK: had primary responsibility for the final content. All authors read and approved the final manuscript and critically reviewed the manuscript.

\section{Conflicts of Interest}

The authors declare no conflict of interest.

\section{References}

1. IARC. Globocan 2012: Estimated Cancer Incidence, Mortality and Prevalence Worldwide in 2012. Available online: http://globocan.iarc.fr/Pages/fact_sheets_cancer.aspx (assessed on 9 June 2014).

2. Jung, K.W.; Won, Y.J.; Kong, H.J.; Oh, C.M.; Lee, D.H.; Lee, J.S. Prediction of Cancer Incidence and Mortality in Korea, 2014. Cancer Res. Treat. 2014, 46, 124-130.

3. Food, Nutrition, Physical Activity, and the Prevention of Cancer: A Global Perspective; World Cancer Research Fund, American Institute for Cancer Research: Washington, DC, USA, 2007.

4. Woo, H.D.; Kim, J. Dietary flavonoid intake and risk of stomach and colorectal cancer. World J. Gastroenterol. 2013, 19, 1011-1019.

5. Zamora-Ros, R.; Agudo, A.; Luján-Barroso, L.; Romieu, I.; Ferrari, P.; Knaze, V.; Bueno-de-Mesquita, H.B.; Leenders, M.; Travis, R.C.; et al. Dietary flavonoid and lignan intake and gastric adenocarcinoma risk in the European Prospective Investigation into Cancer and Nutrition (EPIC) study. Am. J. Clin. Nutr. 2012, 96, 1398-1408.

6. Ekström, A.; Serafini, M.; Nyren, O.; Wolk, A.; Bosetti, C.; Bellocco, R. Dietary quercetin intake and risk of gastric cancer: results from a population-based study in Sweden. Ann. Oncol. 2011, 22, 438-443.

7. Garcia-Closas, R.; Gonzalez, C.A.; Agudo, A.; Riboli, E. Intake of specific carotenoids and flavonoids and the risk of gastric cancer in Spain. Cancer Causes Control. 1999, 10, 71-75.

8. Hara, A.; Sasazuki, S.; Inoue, M.; Iwasaki, M.; Shimazu, T.; Sawada, N.; Yamaji, T.; Tsugane, S. Isoflavone intake and risk of gastric cancer: a population-based prospective cohort study in Japan. Am. J. Clin. Nutr. 2012, 95, 147-154.

9. Rossi, M.; Rosato, V.; Bosetti, C.; Lagiou, P.; Parpinel, M.; Bertuccio, P.; Negri, E.; La Vecchia, C. Flavonoids, proanthocyanidins, and the risk of stomach cancer. Cancer Causes Control. 2010, 21, 1597-1604.

10. Lagiou, P.; Samoli, E.; Lagiou, A.; Peterson, J.; Tzonou, A.; Dwyer, J.; Trichopoulos, D. Flavonoids, vitamin C and adenocarcinoma of the stomach. Cancer Causes Control. 2004, 15, 67-72. 
11. IARC Working Group on the evaluation of carcinogenic risks to humans. Schistosomes, liver flukes and Helicobacter pylori. IARC Monogr. Eval. Carcinog Risks Hum. 1994, 61, 1-241.

12. IARC Working Group on the Evaluation of Carcinogenic Risks to Humans. Tobacco smoke and involuntary smoking. IARC Monogr. Eval. Carcinog. Risks Hum. 2004, 83, 1-1438.

13. Woo, H.D.; Kim, J. Dietary flavonoid intake and smoking-related cancer risk: A meta-analysis. PLoS One 2013, 8, e75604.

14. Vineis, P.; Alavanja, M.; Buffler, P.; Fontham, E.; Franceschi, S.; Gao, Y.T.; Gupta, P.C.; Hackshaw, A.; Matos, E.; Samet, J. Tobacco and cancer: Recent epidemiological evidence. J. Natl. Cancer Inst. 2004, 96, 99-106.

15. Moon, Y.J.; Wang, X.; Morris, M.E. Dietary flavonoids: effects on xenobiotic and carcinogen metabolism. Toxicol. In Vitro 2006, 20, 187-210.

16. Ahn, Y.; Kwon, E.; Shim, J.; Park, M.; Joo, Y.; Kimm, K.; Park, C.; Kim, D. Validation and reproducibility of food frequency questionnaire for Korean genome epidemiologic study. Eur. J. Clin. Nutr. 2007, 61, 1435-1441.

17. Ahn, Y.; Lee, J.E.; Paik, H.Y.; Lee, H.K.; Jo, I.; Kimm, K. Development of a semi-quantitative food frequency questionnaire based on dietary data from the Korea National Health and Nutrition Examination Survey. Nutr. Sci. 2003, 6, 173-184.

18. Yang, Y.K.; Kim, J.Y.; Kwon, O. Development of flavonoid database for commonly consumed foods by Koreans. Korean J. Nutr. 2012, 45, 283-292.

19. Yang, Y.J.; Kim, Y.J.; Yang, Y.K.; Kim, J.Y.; Kwon, O. Dietary flavan-3-ols intake and metabolic syndrome risk in Korean adults. Nutr. Res. Pract. 2012, 6, 68-77.

20. Willett, W. Nutritional Epidemiology; Oxford University Press: New York, NY, USA, 2013.

21. Kim, H.J.; Lim, S.Y.; Lee, J.S.; Park, S.; Shin, A.; Choi, B.Y.; Shimazu, T.; Inoue, M.; Tsugane, S.; Kim, J. Fresh and pickled vegetable consumption and gastric cancer in Japanese and Korean populations: A meta-analysis of observational studies. Cancer Sci. 2010, 101, 508-516.

22. Kim, J.; Kang, M.; Lee, J.S.; Inoue, M.; Sasazuki, S.; Tsugane, S. Fermented and non-fermented soy food consumption and gastric cancer in Japanese and Korean populations: A meta-analysis of observational studies. Cancer Sci. 2011, 102, 231-244.

23. Sasazuki, S.; Tamakoshi, A.; Matsuo, K.; Ito, H.; Wakai, K.; Nagata, C.; Mizoue, T.; Tanaka, K.; Tsuji, I.; Inoue, M. Green tea consumption and gastric cancer risk: an evaluation based on a systematic review of epidemiologic evidence among the Japanese population. Jpn. J. Clin. Oncol. 2012, 42, 335-346.

24. Maselli, A.; Matarrese, P.; Straface, E.; Canu, S.; Franconi, F.; Malorni, W. Cell sex: A new look at cell fate studies. FASEB J. 2009, 23, 978-984.

25. Malorni, W.; Campesi, I.; Straface, E.; Vella, S.; Franconi, F. Redox features of the cell: a gender perspective. Antioxid. Redox Signal. 2007, 9, 1779-1802.

26. Fukai, T.; Marumo, A.; Kaitou, K.; Kanda, T.; Terada, S.; Nomura, T. Anti-Helicobacter pylori flavonoids from licorice extract. Life Sci. 2002, 71, 1449-1463.

27. Ko, K.P.; Park, S.K.; Park, B.; Yang, J.J.; Cho, L.Y.; Kang, C.; Kim, C.S.; Gwack, J.; Shin, A.; Kim, Y. Isoflavones from phytoestrogens and gastric cancer risk: A nested case-control study within the Korean Multicenter Cancer Cohort. Cancer Epidemiol. Biomark. Prev. 2010, 19, 1292-1300. 
28. Yanagihara, K.; Ito, A.; Toge, T.; Numoto, M. Antiproliferative effects of isoflavones on human cancer cell lines established from the gastrointestinal tract. Cancer Res. 1993, 53, 5815-5821.

(C) 2014 by the authors; licensee MDPI, Basel, Switzerland. This article is an open access article distributed under the terms and conditions of the Creative Commons Attribution license (http://creativecommons.org/licenses/by/4.0/). 